\title{
A Case of Thomsen Disease in Pregnancy: Case Report
}

\section{Ayesha Anwar ${ }^{*}$, Adjoa Kyei-Sarpong1, John Obodozie ${ }^{1}$, S. W. Lindow ${ }^{2}$, Ahmed El Sherif ${ }^{1}$, Kashif Ahmad ${ }^{1}$}

${ }^{1}$ North Cumbria University Hospital NHS Trust, Carlisle, UK

${ }^{2}$ Division Obstetrics, Sidra Medical and Research Centre, Al Rayyan, Qatar

Email: *ayeshakashif1@hotmail.com

How to cite this paper: Anwar, A., Kyei-Sarpong, A., Obodozie, J., Lindow, S.W., El Sherif, A. and Ahmad, K. (2019) A Case of Thomsen Disease in Pregnancy: Case Report. Open Journal of Obstetrics and Gynecology, 9, 136-141.

https://doi.org/10.4236/ojog.2019.92014

Received: January 7, 2019

Accepted: January 30, 2019

Published: February 2, 2019

Copyright $\odot 2019$ by author(s) and Scientific Research Publishing Inc. This work is licensed under the Creative Commons Attribution International License (CC BY 4.0).

http://creativecommons.org/licenses/by/4.0/

\begin{abstract}
Thomsen disease is a rare genetic disorder which affects the cell membrane of skeletal muscles causing hyper excitability and periods of prolonged muscle contraction. This prolonged muscle contractions can be aggravated during pregnancy and can interfere with normal labour and delivery. In this case report, we describe the case of a gravid patient in her first pregnancy with Thomsen disease and how to minimise the risk. Also, we illustrate the importance of involving multidisciplinary team in the management of such case to achieve the best fetomaternal outcome.
\end{abstract}

\section{Keywords}

Thomsen Disease, Pregnancy, Anaesthesia

\section{Introduction}

Thomsen disease is an autosomal dominant type of Myotonia Congenita. Myotonia Congenita is a hereditary disorder affecting the tone and contraction of skeletal muscle. The characteristic symptom of Myotonia Congenita is muscle spasm whereby muscles are unable to relax quickly after contracting. The condition is caused by a mutation in the CLCN-1 gene resulting in a faulty synthesis of the proteins that form chloride channels in the skeletal muscle cell membranes, resulting in sustained muscle contraction, stiffness and spasm. Emotional and physical stress can trigger myotonic events.

There are 2 types of inheritance pattern in Myotonia Congenita. The autosomal recessive pattern, or Becker Disease, is the most common and a rare form, Thomsen disease which is autosomal dominant.

In this case report, we present a case of a woman with known Thomsen dis- 
ease during pregnancy and the risks associated with delivery in these patients.

\section{Case}

We report the case of a 29-year-old Caucasian woman who presented in her first pregnancy with a background history of autosomal dominant Myotonia Congenita, also known as Thomsen Disease. She was diagnosed in adulthood following diagnosis of her sister with the same condition. The sisters inherited the condition from their father.

The patient reported mild symptoms that mainly affect the muscles in her hands and face. Specifically, she described a slow-to-relax grip, her jaw occasionally becoming "tight", as well as spasm of her eye muscles and tongue. Her background history included a diagnosis of Irritable Bowel Syndrome for which she was taking Colpermin and Buscopan as well as a Cholecystectomy at the age 16 and wisdom tooth extraction, both performed under general anaesthesia.

We first encountered the patient when she relocated to Cumbria at 37 weeks of gestation and was referred to the Obstetric Medical clinic for review. The patient had experienced an uneventful pregnancy to date, except for episodes of diminished foetal movements from 28 weeks onward. Subsequent growth scans showed a normally grown baby.

An antenatal plan was made regarding labour and delivery. The plan included the need to avoid exposure to low temperature, stress, and dehydration as these could precipitate myotonic spasm. As there are limited information and no available guidance regarding mode of delivery in a pregnant patient with Thomsen disease, a decision of vaginal delivery was discussed and agreed upon with the patient as there are no absolute contraindications for a vaginal birth.

An anaesthetic review showed no airway concerns. The review highlighted a preference for regional anaesthesia over general (although her previous surgeries were under general anaesthesia). If general anaesthesia was unavoidable, it was agreed that the use of Propofol and Neostigmine reversal were to be avoided as these have the potential to cause myotonic spasm. The review also highlighted the need to avoid Suxamethonium, a commonly used muscle relaxant for general anaesthesia, due to the risk of inducing intractable muscle contraction. Alternative options for muscle relaxant included sodium channel blockers such as a local anaesthetic or Dantroline.

Neurology input was requested and consult supported the Obstetric and Anaesthetic plans. Medication was also discussed including the use of Mexiletine, Quinine and carbonic anhydrase inhibitors such as Acetazolamide for management of symptoms. It was agreed that the use of these drugs would only be considered if the benefit clearly outweighed the risks and the patient herself was keen to avoid any medications during pregnancy.

A Paediatric alert was initiated with a plan for review of the baby following delivery.

The patient was then admitted for induction of labour for postdate at a gestation of $41+3$ weeks. A cervical balloon catheter was introduced based on patient 
preference followed by a single $3 \mathrm{mg}$ of Prostin pessary. This was followed by artificial rupture of the membranes and commencement of Oxytocin. Labour was then complicated by abnormal cardiotocography, resulted in category II emergency caesarean section under spinal anaesthesia.

She delivered a live female which required initial resuscitation and was subsequently transferred to the special care baby unit for a brief period of CPAP. As symptoms develop at 3 to 4 years of age, follow up of baby and genetic testing were planned to exclude inheritance of disease.

\section{Discussion}

Myotonia Congenita is a hereditary disorder affecting the tone and contraction of skeletal muscle. It has a worldwide prevalence of 1:23,000 to 1:50,000 [1] although it is more common in Northern Scandinavia where the prevalence is approximately $1: 10,000$.

The primary problem in Myotonia Congenita is inability of skeletal muscle to relax. This is as a result of faulty synthesis of the proteins that form chloride channels in the skeletal muscle cell membranes. Movement of chloride ions across the cell membrane through these channels results in repolarization and muscle relaxation. In Myotonia Congenita faulty transport of chloride ions can sustain prolonged contraction resulting in muscle stiffness and spasm-a characteristic finding of the condition.

The CLCN-1 gene codes for the affected chloride channel and mutation in this gene results in Myotonia Congenita. More than 70 mutations have been identified in the CLCN-1 gene.

There are 2 type of inheritance pattern in Myotonia Congenita. The autosomal recessive pattern, or Becker Disease, is the most common. Both parents of the affected individual are carriers of the mutated gene without showing any sign of the disease. Thomsen Disease is the rarer autosomal dominant type in which only one copy of the altered gene in each cell is sufficient to cause the disorder. In most cases, an affected person has one parent with the condition. The phenotype is typically milder than in Becker Disease [2].

The characteristic symptom of Myotonia Congenita is muscle spasm whereby muscles are unable to relax quickly after contracting. This can cause falls, difficulty in hand shaking and swallowing as well as respiratory difficulties. Individuals with Myotonia Congenita can lead a long, productive life, and can even excel at sports where strength is more important than agility.

In Thomsen Disease, symptoms usually start when the child is 3 to 4 years old, however in the recessive form the onset of symptoms could be delayed. Occasionally in both forms the onset can be as late as 4th or 5th decade of life. Myotonia only occurs when a movement is first initiated. After a few repetitions, the muscle relaxes and the movement becomes normal [3]. This is known as the "warm up phenomenon". Rarely proximal weakness or distal myopathies have been reported in Becker Disease [4]. 
A number of drugs have been used in the treatment of Myotonia Congenita [5]. Mexiletine is a local anaesthetic, antiarrhythmic agent which works by slowing the rate of movement of sodium ions into muscle cells, reducing or eliminating the tendency toward sustained muscle contraction. The use of Quinine is also well documented for both idiopathic leg cramps and the sustained muscle contractions in some myotonic conditions including Myotonia Congenita, with a direct effect on muscle cell membrane sodium channels as the proposed mechanism of action. Acetazolamide-sensitive Myotonia Congenita has been described in the literature.

\section{Pregnancy with Thomsen Disease}

\section{1) Diagnosis and pre conception}

Unless previously diagnosed, diagnosis of Thomsen disease in pregnancy is challenging. There is no single diagnostic test for this condition as there are over 70 mutations in the muscle-chloride muscle gene [6]. Diagnosis of Thomsen disease is usually made based on history, symptoms and signs. In our case diagnosis was made before pregnancy due to family history.

As patients with Myotonia Congenita have an increased risk of adverse anaesthetic-related events, testing of at-risk individuals is reasonable. Pre-pregnancy period is the optimal time to determine genetic risk, classification of carrier status and to discuss the availability of prenatal testing. Therefore, it is appropriate to offer genetic counselling to young couples who are affected, carriers or at risk of being carriers [7]. Genetic counselling should include the potential risks to offspring, different reproductive options and the availability of pre-implantation genetic diagnosis. In our case, the first encounter was at 37 weeks of gestation but a long term plan for neonatal follow up and genetic testing was made.

\section{2) Anaesthesia}

Involvement of anaesthetic opinion is recommended. This should include written plans for the management of labour and in case an emergency caesarean section is required. Epidural and spinal anaesthesia have been used successfully [8]. The highest risk is with general anaesthesia in case of emergency caesarean section. Depolarizing muscle relaxant such as succinylcholine are contraindicated, as they carry a risk of triggering a hyperthermic reaction similar to what is seen in malignant hyperthermia [9]. Moreover, a case report published in 2003, described a serious respiratory complication following administration of suxamethonium to a patient with undiagnosed mytonia congenital [8]. In addition, Propofol was described to cause myotonic crisis [9] [10] and therefore, it should be avoided. Thus, regional anaesthesia is preferred in case of caesarean delivery. The anaesthetic plan in our case was in line with the above.

\section{3) Labour and delivery}

Hormonal effects on chloride channel functions, has been reported to worsen the disease during pregnancy [1]. During pregnancy, some patients experience worsening of symptoms while others remain stable. The first line of treatment is to decrease stress and encourage exercise. For severe symptoms, medical thera- 
pies such as Mexiletine [1] and Acetazolamide [7] are available. Other treatment options include procainamide and quinine [7].

Currently, there are no published guidelines on the management of these cases during pregnancy and delivery, so there is no consensus on the preferred mode of delivery, however both planned caesarean delivery [11] and vaginal birth have been reported [12].

Labour is a known physical and emotional stress which may precipitate a myotonic crisis (muscles enter a state of debilitating contraction and are unable to relax) leading to difficulty in repositioning or pushing during the second stage of labour [5]. However, successful vaginal delivery has been reported [13]. Although, instrumental delivery may help to lessen labour physical stress, there is not enough evidence to support its routine use [5]. Other risk factors for mytonic spasm during labour and delivery include low temperature, use of diathermy and painful intramuscular injections [10]. Management of pregnant patients with Thomsen disease should include a significant amount of robust logistical planning with multidisciplinary involvement. Due to the rarity of these cases, it requires effective communication between different medical teams to avoid detrimental effects on fetomaternal outcome.

\section{Conclusion}

Myotonia Congenita is a rare genetic disorder characterised by myotonic spasms. There is limited data in the literature to guide management in pregnancy and labour; therefore an individualised approach to management should be adopted. Labour and delivery should be guided by the patient's clinical features alongside obstetric considerations. Appropriate multidisciplinary involvement and planning is essential.

\section{Statement}

The patient has given her consent for case report to be published.

\section{Conflicts of Interest}

The authors report no conflict of interest.

\section{References}

[1] Lossin, C. and George, A.L. (2008) Myotonia Congenita. Advances in Genetics, 63, 25-55. https://doi.org/10.1016/S0065-2660(08)01002-X

[2] Colding-Jorgensen, E. (2005) Phenotypic Variability in Myotoniacongenita. Muscle Nerve, 32, 19-34. https://doi.org/10.1002/mus.20295

[3] Sarnat, H.B. (2016) Muscular Dystrophies. In: Kliegman, R.M., Stanton, B.F., St Geme, J.W. and Schor, N.F., Eds., Nelson Textbook of Pediatrics, 20th Edition, Elsevier, Philadelphia, PA, 609.

[4] Nagamitsu, S., Matsuura, T., Khajavi, M., Armstrong, R., Gooch, C., Harati, Y. and Ashizawa, T. (2000) A "Dystrophic" Variant of Autosomal Recessive Myotoniacongenita Caused by Novel Mutations in the CLCN1 Gene. Neurology, 55, 1697-1703. 
https://doi.org/10.1212/WNL.55.11.1697

[5] Yadava, S.M., Garabedian, M., Wallerstein, R.J. and McClellan, L. (2014) Management of Myotonia Congenita in Pregnancy. Journal of Women's Health, Issues \& Care, 3. https://doi.org/10.4172/2325-9795.1000157

[6] Bradley, W.G., Daroff, R.B., Fenichel, G.M. and Jankovic, J. (2008) Neurology in Clinical Practice. Volume II: The Neurological Disorders. 5th Edition, Butterworth Heinemann, Philadelphia.

[7] Duno, M. and Colding-Jorgensen, E. (2011) Myotonia Congenita. GeneReviews, University of Washington, Seattle.

[8] Farbu, E., Softeland, E. and Bindoff, L.A. (2003) Anaesthetic Complications Associated with Myotonia Congenita: Case Study and Comparison with Other Myotonic Disorders. Acta Anaesthesiologica Scandinavica, 47, 630-634. https://doi.org/10.1034/j.1399-6576.2003.00116.x

[9] Bandschapp, O. and Iaizzo, P.A. (2013) Pathophysiologic and Anestheticconsiderations for Patients with Myotoniacongenita or Periodic Paralyses. Pediatric Anesthesia, 23, 824-833.

[10] Gorthi, S., Radbourne, S., Drury, N. and Rajagopalan, C. (2013) Management of Pregnancy with Thomsen's Disease. European Journal of Obstetrics Gynecology and Reproductive Biology, 170, 293-296.

https://doi.org/10.1016/j.ejogrb.2013.05.013

[11] Boyle, R. (1999) Antenatal and Preoperative Genetic and Clinical Assessment in Myotonic Dystrophy. Anaesthesia and Intensive Care Journal, 27, 301-306. https://doi.org/10.1177/0310057X9902700315

[12] Basu, A., Nishanth, P. and Ifaturoti, O. (2009) Pregnancy in Women with Myotonia Congenita. International Journal of Gynecology \& Obstetrics, 106, 62-63. https://doi.org/10.1016/j.ijgo.2009.01.031

[13] Farrow, C. and Carling, A. (2007) Successful Spinal Blockade in a Parturient with Myotonia Congenita. International Journal of Obstetric Anesthesia, 16, 89-90. https://doi.org/10.1016/j.ijoa.2006.06.011 